\title{
A Simple Method for Finding Emperical Liklihood Type Intervals for the ROC Curve
}

\author{
Ayman Baklizi \\ QatarUniversity, a.baklizi@qu.edu.qa
}

Follow this and additional works at: http://digitalcommons.wayne.edu/jmasm

Part of the Applied Statistics Commons, Social and Behavioral Sciences Commons, and the Statistical Theory Commons

\section{Recommended Citation}

Baklizi, Ayman (2007) "A Simple Method for Finding Emperical Liklihood Type Intervals for the ROC Curve," Journal of Modern Applied Statistical Methods: Vol. 6 : Iss. 2 , Article 23.

DOI: $10.22237 /$ jmasm/1193890920

Available at: http://digitalcommons.wayne.edu/jmasm/vol6/iss2/23 


\title{
A Simple Method For Finding Empirical Likelihood Type Intervals For The ROC Curve
}

\author{
Ayman Baklizi \\ Qatar University
}

Interval estimation of the ROC curve is considered using the empirical likelihood techniques. Suggested is a procedure that is very simple computationally and avoids the constrained optimization problems usually faced with empirical likelihood methods. Various modifications are suggested and the performance of the intervals is evaluated in terms of their coverage probability. The results show tat some of the suggested intervals compete well with other intervals known in the literature.

Key words: ROC curve, empirical likelihood, kernel estimators, bootstrap

\section{Introduction}

The Receiver Operating Characteristic (ROC) curve is used to assess the accuracy of a diagnostic test in discriminating between healthy and diseased individuals. A threshold value $c$ is determined, and people with test measurements greater than $c$ are classified as diseased, otherwise as healthy. Let $X$ be a random variable representing the test score of a healthy individual and let $Y$ be the score of a diseased patients. Let $F$ and $G$ be the distribution functions of $X$ and $Y$ respectively. The sensitivity of the test is defined as $1-G(c)$. It is the probability that the test score of diseased patient is greater than $c$. The specificity of the test is defined as $F(c)$, it is the probability of correctly classifying a healthy individual. The receiver operating characteristic curve is defined as the plot of $1-F(c)$ against $1-G(c)$ as $c$ varies from $-\infty$ to $\infty$ or equivalently as the plot of $1-G\left(F^{-1}(1-t)\right)$ where $0 \leq t \leq 1$, (Hsieh and Turnbull, 1996).

Ayman Baklizi is an Associate Professor of statistics, and is at the department of mathematics and physics at Qatar University. $\mathrm{He}$ is an elected-member at the ISI. Email: baklizi1@yahoo.com
The estimation of the ROC curve has received considerable attention. The problem has been considered in parametric, nonparametric and semi-parametric situations. For example, see Hsieh and Turnbull (1996), Li et al. (1999), Hall et al., (2003).

Claeskens et al. (2003) developed empirical likelihood confidence regions for the ROC curve. Let $X_{1}, \ldots, X_{n}$ and $Y_{1}, \ldots, Y_{m}$ be two random samples from the distributions $F$ and $G$ respectively. Define the ROC curve as $R(t)=1-G\left(F^{-1}(1-t)\right)$ where $0 \leq t \leq 1$ and let $\theta=R(t)$, Claeskens et al. (2003) constructed confidence intervals for $\theta$ using the smoothed empirical likelihood function

$$
L(\theta)=\sup _{(p, q, \eta)}\left(\prod_{i=1}^{n} p_{i}\right)\left(\prod_{j=1}^{m} q_{j}\right),
$$

where $p^{\prime}=\left(p_{1}, \ldots, p_{n}\right)$ and $q^{\prime}=\left(q_{1}, \ldots, q_{m}\right)$ are probability vectors each summing to one and subject to certain constraints on the smoothed versions of the empirical distributions of $X$ and $Y$. They showed that the asymptotic distribution of the $\log$-likelihood ratio $l(\theta)=-2 \log L(\theta)$ is chi square with one degree of freedom and conducted some simulations to investigate the performance of their intervals and show that it performs better than some other asymptotic and bootstrap intervals. 


\section{Purpose}

An alternative procedure is suggested here based on the empirical likelihood which is very simple computationally, does not need numerical constrained optimization, and produces interval estimates that are, in some cases, about as accurate as those of Claeskens et al. (2003). This procedure and some modifications are described. A simulation experiment was conducted to investigate and compare the suggested procedure with other well known procedures.

Empirical Likelihood Based Intervals

Assume that an interval estimator of $R\left(t^{*}\right)=1-G\left(F^{-1}\left(1-t^{*}\right)\right)$ is desired where $t^{*}$ is some specific point in the unit interval. Proceed in two stages as follows; in the first stage obtain a point estimator for $F^{-1}\left(1-t^{*}\right)$. This is equivalent to estimating $x_{1-t^{*}}:\left(1-t^{*}\right)^{t h}$ quantile of $F$ denote this estimator by $\hat{x}_{1-t^{*}}$. In the second stage obtain an interval estimator of $\bar{G}\left(\hat{x}_{1-t^{*}}\right)=1-G\left(\hat{x}_{1-t^{*}}\right)$ which is the right tail probability of the random variable $Y$ having distribution function $G$.

In an empirical likelihood setup, the first stage amounts to estimating $x_{1-t^{*}}$ which may be done using interpolation between the values of the ordered statistics of the sample of the distribution of $X$. In the second stage consider the empirical likelihood function for quantiles (Owen, 2001) given by

$$
\begin{aligned}
& R(p, q)= \\
& \max \left\{\prod_{i=1}^{m} n w_{i} \mid \sum_{i=0}^{m+1} w_{i} Z_{i}(p, q)=0, w_{i} \geq 0, \sum_{i=0}^{m+1} w_{i}=1\right\}
\end{aligned}
$$

where $0 \leq p \leq 1, \quad-\infty<q<\infty$ is the $p^{\text {th }}$ quantile and $Z_{i}(p, q)=I_{\left(X_{i} \leq q\right)}-p$. Substituting $\hat{x}_{1-t^{*}}$ for $q$ and $G\left(\hat{x}_{1-t^{*}}\right)$ for $p$ and, conditional on $\hat{x}_{1-t^{*}}$ using the empirical likelihood function $R\left(G\left(\hat{x}_{1-t^{*}}\right), \hat{x}_{1-t^{*}}\right)$ one can construct confidence interval for $G\left(\hat{x}_{1-t^{*}}\right)$ as

$$
\left\{G\left(\hat{x}_{1-t^{*}}\right) \mid-2 \log R\left(G\left(\hat{x}_{1-t^{*}}\right), \hat{x}_{1-t^{*}}\right)>\chi_{\alpha, 1}^{2}\right\}
$$

and then transform it to a confidence interval for $1-G\left(\hat{x}_{1-t^{*}}\right)$ this results in a confidence interval for $R\left(t^{*}\right)$ Call this interval the (EL) interval.

The chi square calibration used in the empirical likelihood interval may be replaced by the E-Calibration of Tsao (2004). This calibration is based on the quantiles of a new family of distributions arising from the normal distribution. It is derived using the finite sample similarity between the empirical and parametric likelihoods. Some quantiles $e_{\alpha, 1, m}$ of that distribution are given in Tsao (2004). The E calibration corrects for under coverage resulting from using the chi square calibration. The new interval (EC interval) based on this calibration is given by

$$
\left\{G\left(\hat{x}_{1-t^{*}}\right) \mid-2 \log R\left(G\left(\hat{x}_{1-t^{*}}\right), \hat{x}_{1-t^{*}}\right)>e_{\alpha, 1, m}\right\}
$$

Another modification may be obtained by using the "smoother" version of the empirical likelihood function for quantiles introduced by Adimari (1998). In this modification the empirical likelihood is replaced by a smoother version which, when considered as a function of $G\left(\hat{x}_{1-t^{*}}\right)$, may be written as

$$
\begin{aligned}
& -2 \log \tilde{R}\left(G\left(\hat{x}_{1-t^{*}}\right), \hat{x}_{1-t^{*}}\right)= \\
& 2 \tilde{G}\left(\hat{x}_{1-t^{*}}\right) \log \left(\frac{\tilde{G}\left(\hat{x}_{1-t^{*}}\right)}{G\left(\hat{x}_{1-t^{*}}\right)}\right)+ \\
& \left(1-\tilde{G}\left(\hat{x}_{1-t^{*}}\right) \log \left(\frac{1-\tilde{G}\left(\hat{x}_{1-t^{*}}\right)}{1-G\left(\hat{x}_{1-t^{*}}\right)}\right)\right)
\end{aligned}
$$

where

$$
\widetilde{G}\left(\hat{x}_{1-t^{*}}\right)= \begin{cases}G^{*}\left(\hat{x}_{1-t^{*}}\right) & \text { if }\left[\mathrm{Y}_{(1)}, Y_{(m)}\right] \text { contains } \hat{x}_{1-t^{*}} \\ \hat{G}\left(\hat{x}_{1-t^{*}}\right) & \text { otherwise }\end{cases}
$$

where $G^{*}=\frac{2 i-1}{2 n}$ on each $Y_{(i)}$ and is linear in each $\left\lfloor Y_{(i)}, Y_{(i+1)}\right\rfloor$, and where $Y_{(1)}, \ldots, Y_{(n)}$ are the 
order statistics of the sample of $Y$ values. Adimari showed that the limiting distribution is also $\chi_{1}^{2}$. A $(1-\alpha) \%$ confidence interval for $G\left(\hat{x}_{1-t^{*}}\right)$ (AD interval) is given by

$$
\left\{G\left(\hat{x}_{1-t^{*}}\right) \mid-2 \log \widetilde{R}\left(G\left(\hat{x}_{1-t^{*}}\right), \hat{x}_{1-t^{*}}\right)>\chi_{1, \alpha}^{2}\right\}
$$

Simulation

Simulation studies were conducted to assess the performance of the interval estimates based in the empirical likelihood. Also considered were the bootstrapped version of the empirical likelihood interval (BEL), and the bootstrapped version of the (AD) interval, the (BTAD) interval. A Bartlett type correction factor is obtained as the mean of the B bootstrap empirical log-likelihood ratios which in turn used to find the (BRT) interval. The simulation design used similar to those used by Claeskens et al.(2003) and Hall et. al. (2003). The coverage probability were investigated at values of $t=$ $0.1,0.3,0.5,0.7$ and 0.9 with sample size $(n, m)=(30,30),(50,50),(70,70),(100,100)$, $(50,70)$ and $(70,50)$. In each case 2000 pair of samples is generated from

$$
\begin{gathered}
\text { 1- } X \sim N(0,1), Y \sim N(1,1) \\
\text { 2- } X \sim \Gamma(2), Y \sim \Gamma(3) \\
\text { 3- } X \sim t(5), \\
Y \sim 0.2(t(5)-1)+0.8(t(5)-1)
\end{gathered}
$$

$\mathrm{B}=500$ is used in bootstrap calculations. The coverage probabilities of the intervals with nominal confidence levels $(1-\alpha)=0.90$ and 0.95 are given in Tables 1-3.

\section{Result}

The results are given in tables $1-3$ where the following abbreviations are used EL: The empirical likelihood interval based on the asymptotic $\chi^{2}$ approximation. BEL: The empirical likelihood interval based on bootstrap crirical values.

EC: The empirical likelihood interval based on Tsao's E-Calibration. BRT: The empirical likelihood interval with the bootstrap Bartlett type correction. AD: The empirical likelihood interval based on Adimari's modification.
BTAD: The empirical likelihood interval based on Adimari's modification and bootstrap critical values.

\section{Conclusion}

It appears that the coverage probabilities of the intervals are close to the nominals for small values of $t$. For larger values of $t$ most intervals tend to have an undercoverage problem. Exceptions are the bootstrapped empirical likelihood interval (BEL) and the corrected interval (BRT). These two intervals tend to be conservative for larger values of $t$. A drawback of the (BEL) interval is that it has a very low coverage probability for small values of $t$ when the sample sizes differ.

This is not the case with the (BRT) interval. These observations are also applicable to the results given in tables 2 and 3. The BRT in most cases have the closest coverage probability to nominal. Comparison of these results with Hall et. al. (2003) and Claeskens et al. (2003) shows that the (BRT) interval considered in this article competes very well with theirs in terms of its coverage probability. The simplicity of the methods discussed in this article and the avoidance of complicated restricted optimization problems or sophisticated bandwidth rules used for the construction of kernel based intervals may balance the slightly better performance of the Hall et al. (2003) or Claeskens et. al. (2003) intervals.

\section{References}

Adimari, G. (1998). An empirical likelihood statistic for quantiles. Journal of Statistical Computation and Simulation, 60, 85 95.

Hall, P., Hyndman, R. \& Fan, Y. (2003). Nonparametric confidence intervals for receiver operating characteristic curves. Biometrika, 91, $3,743-750$.

Claeskens, G., Jing, B. Peng, L., \& Zhou, W. (2003). Empirical likelihood confidence regions for comparison distributions and ROC curves. The Canadian Journal of Statistics, 31, 173 - 190. 
Table 1. Coverage Probabilities of the Intervals, The Normal Distribution

\begin{tabular}{|c|c|c|c|c|c|c|c|c|c|c|c|c|c|c|}
\hline \multirow[t]{2}{*}{$n$} & \multirow[t]{2}{*}{$m$} & \multirow[t]{2}{*}{$t$} & \multirow{2}{*}{\multicolumn{4}{|c|}{$\begin{array}{l}\text { EC } \quad \text { BRT } \\
\alpha=0.10\end{array}$}} & $A D$ & BTAD & EL & BEL & $\mathrm{EC}$ & BRT & $A D$ & BTAD \\
\hline & & & & & & & & & \multicolumn{6}{|c|}{$\alpha=0.05$} \\
\hline \multirow[t]{5}{*}{30} & 30 & 0.1 & 887 & 182 & 887 & 791 & 926 & 932 & 887 & 228 & 967 & 900 & 951 & 961 \\
\hline & & 0.3 & 743 & 741 & 743 & 779 & 761 & 768 & 794 & 791 & 946 & 803 & 789 & 947 \\
\hline & & 0.5 & 816 & 852 & 816 & 872 & 805 & 817 & 864 & 903 & 864 & 906 & 877 & 899 \\
\hline & & 0.7 & 719 & 881 & 743 & 903 & 767 & 789 & 840 & 928 & 840 & 931 & 848 & 863 \\
\hline & & 0.9 & 600 & 839 & 600 & 876 & 676 & 695 & 623 & 921 & 705 & 917 & 768 & 788 \\
\hline \multirow[t]{5}{*}{50} & 50 & 0.1 & 927 & 378 & 927 & 878 & 920 & 925 & 927 & 411 & 927 & 917 & 951 & 54 \\
\hline & & 0.3 & 852 & 809 & 852 & 824 & 848 & 855 & 905 & 864 & 905 & 872 & 905 & 906 \\
\hline & & 0.5 & 757 & 906 & 819 & 926 & 803 & 811 & 868 & 951 & 868 & 953 & 874 & 884 \\
\hline & & 0.7 & 729 & 914 & 729 & 950 & 746 & 756 & 811 & 965 & 811 & 966 & 832 & 841 \\
\hline & & 0.9 & 651 & 890 & 651 & 948 & 692 & 700 & 731 & 965 & 731 & 971 & 783 & 791 \\
\hline \multirow[t]{5}{*}{70} & 70 & 0.1 & 905 & 531 & 905 & 927 & 936 & 937 & 966 & 561 & 966 & 956 & 960 & 961 \\
\hline & & 0.3 & 855 & 874 & 855 & 890 & 836 & 840 & 855 & 909 & 892 & 919 & 922 & 925 \\
\hline & & 0.5 & 783 & 924 & 783 & 952 & 790 & 797 & 860 & 961 & 860 & 971 & 866 & 870 \\
\hline & & 0.7 & 756 & 932 & 756 & 971 & 761 & 765 & 838 & 978 & 838 & 984 & 838 & 844 \\
\hline & & 0.9 & 662 & 907 & 662 & 972 & 692 & 694 & 732 & 974 & 732 & 984 & 773 & 777 \\
\hline \multirow[t]{5}{*}{100} & 100 & 0.1 & 941 & 658 & 941 & 945 & 938 & 938 & 941 & 677 & 941 & 970 & 967 & 967 \\
\hline & & 0.3 & 830 & 895 & 830 & 918 & 833 & 833 & 880 & 940 & 880 & 941 & 906 & 906 \\
\hline & & 0.5 & 800 & 946 & 800 & 978 & 803 & 803 & 868 & 978 & 868 & 986 & 882 & 882 \\
\hline & & 0.7 & 719 & 945 & 719 & 986 & 743 & 743 & 832 & 988 & 832 & 992 & 832 & 832 \\
\hline & & 0.9 & 646 & 929 & 646 & 980 & 661 & 661 & 722 & 980 & 722 & 991 & 752 & 752 \\
\hline \multirow[t]{5}{*}{50} & 70 & 0.1 & 878 & 506 & 878 & 901 & 918 & 922 & 947 & 540 & 947 & 939 & 946 & 948 \\
\hline & & 0.3 & 815 & 847 & 815 & 867 & 812 & 813 & 815 & 889 & 859 & 899 & 902 & 907 \\
\hline & & 0.5 & 747 & 910 & 747 & 943 & 763 & 768 & 833 & 959 & 833 & 959 & 836 & 842 \\
\hline & & 0.7 & 708 & 911 & 708 & 958 & 715 & 724 & 791 & 973 & 791 & 978 & 800 & 800 \\
\hline & & 0.9 & 587 & 885 & 587 & 946 & 610 & 620 & 646 & 962 & 646 & 971 & 694 & 701 \\
\hline \multirow[t]{5}{*}{70} & 50 & 0.1 & 958 & 397 & 958 & 924 & 952 & 954 & 958 & 435 & 958 & 953 & 972 & 974 \\
\hline & & 0.3 & 869 & 815 & 869 & 823 & 864 & 870 & 912 & 869 & 912 & 875 & 907 & 911 \\
\hline & & 0.5 & 780 & 909 & 845 & 925 & 823 & 835 & 881 & 954 & 881 & 951 & 890 & 899 \\
\hline & & 0.7 & 791 & 928 & 791 & 962 & 789 & 797 & 871 & 976 & 871 & 979 & 867 & 874 \\
\hline & & 0.9 & 710 & 910 & 710 & 957 & 735 & 743 & 786 & 965 & 786 & 976 & 812 & 818 \\
\hline
\end{tabular}


Table 2. Coverage Probabilities of the Intervals, Asymmetric Distributions Case

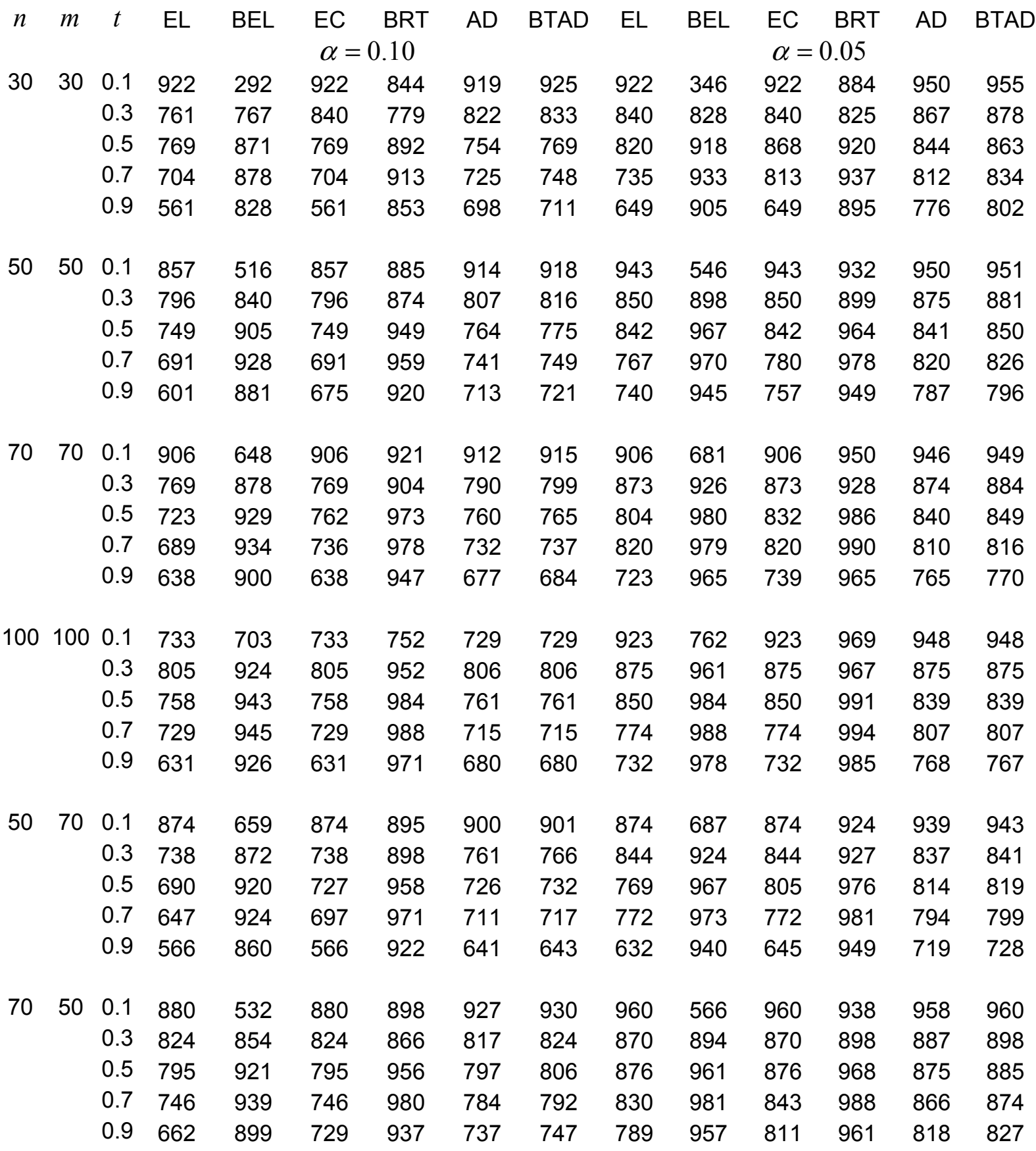


Table 3: Coverage Probabilities of the Intervals, Mixture Distributions Case

\begin{tabular}{|c|c|c|c|c|c|c|c|c|c|c|c|c|c|c|}
\hline$n$ & $m$ & $t$ & EL & BEL & $\begin{array}{c}\mathrm{EC} \\
\alpha=\end{array}$ & $\begin{array}{l}\text { BRT } \\
.10\end{array}$ & $A D$ & BTAD & EL & BEL & $\begin{array}{l}\mathrm{EC} \\
\alpha=\end{array}$ & $\begin{array}{l}\text { BRT } \\
0.05\end{array}$ & $A D$ & BTAD \\
\hline \multirow[t]{5}{*}{30} & 30 & 0.1 & 808 & 792 & 808 & 804 & 844 & 852 & 861 & 850 & 861 & 844 & 873 & 879 \\
\hline & & 0.3 & 820 & 899 & 877 & 921 & 846 & 858 & 877 & 938 & 877 & 949 & 912 & 30 \\
\hline & & 0.5 & 828 & 927 & 828 & 956 & 854 & 874 & 914 & 964 & 914 & 972 & 916 & 928 \\
\hline & & 0.7 & 755 & 903 & 755 & 937 & 792 & 809 & 825 & 960 & 842 & 961 & 864 & 384 \\
\hline & & 0.9 & 590 & 824 & 590 & 851 & 678 & 692 & 699 & 906 & 699 & 906 & 764 & 783 \\
\hline \multirow[t]{5}{*}{50} & 50 & 0.1 & 838 & 866 & 838 & 886 & 826 & 834 & 838 & 902 & 838 & 917 & 907 & 10 \\
\hline & & 0.3 & 863 & 934 & 863 & 962 & 842 & 848 & 895 & 968 & 927 & 971 & 907 & 14 \\
\hline & & 0.5 & 811 & 952 & 851 & 984 & 846 & 853 & 911 & 985 & 911 & 990 & 909 & 16 \\
\hline & & 0.7 & 813 & 937 & 813 & 977 & 798 & 807 & 879 & 978 & 879 & 986 & 869 & 16 \\
\hline & & 0.9 & 595 & 880 & 595 & 919 & 668 & 679 & 685 & 946 & 685 & 951 & 749 & 60 \\
\hline \multirow[t]{5}{*}{70} & 70 & 0.1 & 828 & 887 & 828 & 906 & 815 & 820 & 867 & 928 & 867 & 939 & 891 & 90 \\
\hline & & 0.3 & 841 & 951 & 881 & 984 & 852 & 857 & 908 & 982 & 908 & 988 & 917 & 21 \\
\hline & & 0.5 & 837 & 956 & 837 & 988 & 830 & 836 & 893 & 987 & 893 & 994 & 901 & 905 \\
\hline & & 0.7 & 807 & 962 & 807 & 990 & 803 & 808 & 872 & 991 & 885 & 995 & 880 & 382 \\
\hline & & 0.9 & 604 & 907 & 662 & 957 & 663 & 672 & 734 & 968 & 734 & 974 & 752 & 62 \\
\hline \multirow[t]{5}{*}{100} & 100 & 0.1 & 787 & 319 & 787 & 941 & 811 & 811 & 873 & 959 & 873 & 959 & 889 & \\
\hline & & 0.3 & 843 & 965 & 843 & 991 & 853 & 853 & 924 & 989 & 924 & 997 & 923 & 923 \\
\hline & & 0.5 & 834 & 970 & 834 & 996 & 843 & 843 & 899 & 995 & 899 & 998 & 907 & 907 \\
\hline & & 0.7 & 775 & 967 & 775 & 996 & 799 & 799 & 873 & 993 & 873 & 998 & 868 & 868 \\
\hline & & 0.9 & 634 & 908 & 634 & 968 & 662 & 662 & 686 & 977 & 686 & 986 & 746 & 74 \\
\hline \multirow[t]{5}{*}{50} & 70 & 0.1 & 780 & 858 & 780 & 887 & 781 & 786 & 827 & 908 & 827 & 922 & 858 & 361 \\
\hline & & 0.3 & 832 & 949 & 871 & 977 & 840 & 846 & 894 & 981 & 894 & 986 & 900 & 904 \\
\hline & & 0.5 & 820 & 964 & 820 & 992 & 810 & 815 & 875 & 990 & 875 & 997 & 882 & 886 \\
\hline & & 0.7 & 766 & 943 & 766 & 979 & 760 & 767 & 819 & 981 & 836 & 990 & 841 & 846 \\
\hline & & 0.9 & 521 & 870 & 570 & 929 & 600 & 605 & 638 & 946 & 638 & 956 & 696 & 702 \\
\hline \multirow[t]{5}{*}{70} & 50 & 0.1 & 859 & 869 & 859 & 884 & 839 & 851 & 859 & 903 & 859 & 911 & 918 & 92 \\
\hline & & 0.3 & 886 & 940 & 886 & 965 & 867 & 871 & 912 & 975 & 946 & 979 & 931 & 938 \\
\hline & & 0.5 & 818 & 962 & 853 & 980 & 847 & 853 & 920 & 985 & 920 & 990 & 915 & 922 \\
\hline & & 0.7 & 834 & 942 & 834 & 983 & 824 & 835 & 907 & 983 & 907 & 990 & 897 & 90 \\
\hline & & 0.9 & 656 & 889 & 656 & 949 & 715 & 724 & 733 & 958 & 733 & 971 & 793 & 80 \\
\hline
\end{tabular}


Hsieh, F., \& Turnbull, B. (1996). Nonparametric and semiparametric estimation of the receiver operating characteristic curve. The Annals of Statistics, 24, 1, 25 - 40.

Li, G., Tiwari, R., \& Wells, M. (1999). Semiparametric inference for a quantile comparison function with applications to receiver operating characteristic curve. Biometrika, 86, 3, 487 - 502.
Owen, A. B. (2001). Empirical Likelihood. NY: Chapman and Hall.

Tsao, M. (2004). A new method of calibration for the empirical loglikelihood ratio. Statistics and Probability Letters, 68, 305 - 314. 